\title{
INVESTIGACIÓN COMPARATIVA SOBRE LAS EVALUACIONES DE ESTUDIANTES UNIVERSITARIOS FRENTE A DOS MODELOS E-LEARNING
}

\author{
Andrés Mendiburo-Seguel ${ }^{1}$, Marion Reininger ${ }^{2}$
}

\section{RESUMEN}

Este artículo resume los resultados de una investigación realizada en una universidad chilena, cuyo fin fue comparar las evaluaciones de dos grupos de estudiantes frente a dos modelos de e-learning. Ambos se diferencian respecto a múltiples factores. Se explican los modelos, así como también la revisión teórica que llevó a la creación del instrumento para evaluar dichos factores. Se presentan luego los resultados, destacando que al comparar las evaluaciones de los diferentes factores de los dos modelos de e-learning, los alumnos que siguieron estudios a través de la modalidad recientemente implementada tienen una evaluación significativamente más alta del respectivo sistema, que aquellos que estudiaron bajo la modalidad anterior. Finalmente se discuten las implicancias de estos resultados.

Palabras clave: e-learning, modelos educativos e-learning, evaluación e-learning, factores de evaluación e-learning

\section{A COMPARATIVE REASEARCH ABOUT EVALUATIONS UNIVERSITY STUDENTS MADE ON TWO MODELS OF E-LEARNING}

\section{ABSTRACT}

This article summarizes the outcomes from a research carried out by a Chilean university aimed to compare the evaluations two groups of students made on two different e-learning models. These models differ in multiple factors. However both are explained, as well as the theoretical review leading to the creation of an instrument to assess such factors. Results show that when comparing the evaluations of the different factors of the two e-learning models, learners from the newly implemented e-learning model have a significantly higher evaluation of the relevant system than those who studied under the previous model. Finally, the implications of these results are discussed.

Keywords: e-learning, e-learning education models, e-learning evaluation, e-learning evaluation factors

1 Investigador senior, Centro de Estudios Universitarios Universidad UNIACC, Santiago, Chile.

2 Coordinadora académica de Prorrectoría. Universidad UNIACC, Santiago, Chile.

Contacto: andres.mendiburo@uniacc.cl 


\section{INVESTIGACIÓN COMPARATIVA SOBRE LAS EVALUACIONES DE ESTUDIANTES UNIVERSITARIOS FRENTE A DOS MODELOS E-LEARNING}

\section{Introducción}

Desde el año 2000 se ha podido observar la incorporación de las tecnologías de la información y comunicación en la educación superior chilena. El uso de herramientas digitales como una forma de apoyar la enseñanza se ha extendido, pero sólo un porcentaje limitado de los contenidos se provee de forma online y ellos están generalmente relacionados con los aspectos administrativos de la enseñanza. Esto significa que la educación virtual en Chile es ante todo entendida como un mecanismo complementario de la formación, conocido como eSupport.

El porcentaje limitado de los contenidos que se proveían online, ha evolucionado progresivamente a modelos como el blended learning (modelos mixtos de educación) o el e-learning. Considerando que dichos modelos se han adaptado en mayor medida en la universidad (Universidad Virtual-REUNA, 2003), gradualmente se ha observado que distintos programas que involucran entrega de grados académicos se han abierto a estas modalidades. Sin embargo, siguen siendo un porcentaje menor dentro del contexto. De acuerdo con Riquelme y Martínez (2008), el sistema educacional online chileno ofrece cursos generales en un $55 \%$, diplomados en un $27 \%$, programas de pregrado en un $9 \%$, programas de maestría en un $8 \%$, y programas doctorales en un $1 \%$.

Pero, ¿qué significa que un programa sea provisto a distancia, de forma online, virtual, o como lo denominaremos en este estudio: e-learning? En las diversas conceptualizaciones existentes, tienden a concurrir cuatro elementos, que Simonson, Smaldino, Albright y Zvacek (2006, p. 7) describen de la siguiente manera: 
- Consiste en un proceso de educación formal, que posee una base institucional

- Se expresa en la separación del profesor y el alumno

- Utiliza sistemas de telecomunicación interactivos

- Conecta al alumno, al profesor y los recursos de aprendizaje, compartiendo información, datos, entre otros, por medio de distintos formatos que integran la experiencia educativa.

En este sentido, el e-learning posee los mismos fines que son transversales a todo proceso de enseñanza-aprendizaje, esto es, el desarrollo y/o perfeccionamiento de conocimientos y habilidades, lo cual fundamenta su consecuente incorporación a las distintas casas de estudio. Su particularidad se encuentra en la no copresencialidad entre el educador y el educando, donde todos o la mayor parte de los contenidos son provistos online, lo que se cuantifica como un $80 \%$ de dichos contenidos y más entregados a distancia, comúnmente no contemplando encuentros cara a cara (Allen y Seaman, 2009). Esta separación física se ve compensada con mecanismos de vinculación de las partes involucradas, entre sí y con los contenidos definidos, por medio de la utilización de modelos pedagógicos y tecnológicos acordes. Los sistemas de telecomunicación permiten la interacción mediante actividades sincrónicas y asincrónicas que posibilitan la generación de un espacio educativo a distancia.

El presente estudio aborda el tema del e-learning desde la perspectiva evaluativa de los alumnos que cursan diferentes programas de pre y posgrado. Se consideraron las evaluaciones de estudiantes con respecto a diferentes factores levantados teóricamente de la información disponible, a través de una escala ad hoc. Se compararon las evaluaciones, pues se sospechó que el actual modelo (PMO) sería mejor evaluado por los estudiantes que el antiguo modelo (PET), toda vez que tiene una estructura de creación y entrega online más acorde a las necesidades actuales.

El artículo comienza explicando las dos modalidades de e-learning que se compararon. Luego se revisa la teoría existente respecto a los factores a evaluar de un programa online, para finalmente mostrar los resultados y conclusiones de la investigación. 


\section{Caracterización de los modelos e-learning}

\subsection{Programa Especial de Titulación Online, PET}

Es un sistema académico-administrativo que permite proveer programas a distancia bajo la modalidad e-learning en una estrategia que coordina el proceso de enseñanza-aprendizaje asincrónico. Éste se focaliza en la publicación sistemática de contenidos, apoyados por material multimedia, sobre los cuales el alumno debe basar su participación por escrito en la plataforma virtual, acción que es orientada y evaluada por un profesor. Este programa se implementó en Universidad UNIACC el 2004, siendo ésta la primera institución en Chile que ofrecía carreras de pregrado 100\% online en dicho momento, incluyendo posteriormente titulación de posgrado.

El PET Online se basa en un modelo centrado en el profesor como pilar del proceso educativo, quien provee el material de estudio, principalmente constituido por textos de su autoría y alusión a otros referentes de la disciplina; define la metodología utilizada en el desarrollo didáctico asociada a la transmisión de dichos contenidos y a la evaluación de la adquisición de los mismos, así como el número y tipo de evaluaciones adecuadas para el éxito formativo del alumno. El momento en que se llevaban a cabo las evaluaciones dependía también del profesor. Puede considerarse que el método de PET se acerca más a la idea de enseñanza clásica, pero a través de plataformas con base en internet ${ }^{3}$.

Los cursos de pregrado a través del sistema PET duraban catorce semanas, incluyendo exámenes de repetición, y en promedio se dictaban seis paralelamente. Se exigían dos evaluaciones por cátedra, aún cuando algunas carreras, como psicología, involucraban una tercera que era resultado del promedio de trabajos grupales.

En el posgrado, los cursos a través del sistema PET duraban trece semanas, en las cuales se dictaban dos cursos obligatorios de la

3 Director del Programa Modular Online UNIACC, entrevista personal, 17 de marzo de 2011. 
malla y uno electivo. Las evaluaciones funcionaban del mismo modo que en el caso de pregrado ${ }^{4}$.

Con respecto al número de horas por cada asignatura, éstas podían variar entre tres y cinco, no pudiendo excederse las veinte horas. A excepción de ingeniería informática, la cual consideraba cincuenta y dos horas fijas para cada curso en el semestre.

Los cursos funcionaban como prerrequisito de otros, según la construcción de cada malla curricular.

Con la utilización de Moodle, como la plataforma digital que soporta al PET Online, se logra el traslado de la cátedra a contenidos y presentaciones en línea; de la comunicación presencial a la interacción vía email, foros, entre otros recursos digitales que posibilitaron el paso de la modalidad presencial a la de e-learning (Cabrera, 2009).

Gráfico n. ${ }^{\circ}$ 1: Estructura curricular del modelo PET Online

\begin{tabular}{|c|c|c|c|}
\hline $\begin{array}{l}\text { imestre } 1 \\
2 \text { semanas }\end{array}$ & $\begin{array}{l}\text { Trimestre } 2 \\
12 \text { semanas }\end{array}$ & $\begin{array}{l}\text { Trimestre } 3 \\
12 \text { semanas }\end{array}$ & $\begin{array}{l}\text { Trimestres } \\
\text { sucesivos }\end{array}$ \\
\hline Asignatura 1 & Asignatura 6 & Asignatura & \multirow{5}{*}{$\begin{array}{c}\text { Hasta concluir } \\
\text { la totalidad } \\
\text { de trimestres } \\
\text { establecidos para } \\
\text { cada carrera de pre y } \\
\text { posgrado }\end{array}$} \\
\hline Asignatura 2 & Asignatura 7 & Asignatura & \\
\hline Asignatura 3 & Asignatura 8 & Asignatura & \\
\hline Asignatura 4 & Asignatura 9 & Asignatura & \\
\hline Asignatura 5 & Asignatura 10 & Asignatura & \\
\hline
\end{tabular}

\subsection{Programa Modular Online, $\mathrm{PMO}$}

Es un sistema académico-administrativo que posibilita el desarrollo de programas e-learning mediante una nueva estrategia didáctica implementada gradualmente en la universidad UNIACC desde 2008 en las carreras de pre y posgrado. El enfoque pedagógico que sustenta este modelo se asocia a la taxonomía de objetivos educativos de Bloom. Este modelo se entiende como la relación entre un estímulo formativo y una respuesta a éste, en términos de un aprendizaje observable que alcance las metas establecidas. Dicho estímulo se orienta a la

4 Director la Escuela de Posgrado UNIACC, entrevista personal, 18 de mayo de 2011. 

UNIVERSITARIOS FRENTE A DOS MODELOS E-LEARNING - A. Mendiburo-Seguel, M. Reininger

consecución de alguno de los seis niveles cognitivos establecidos en la taxonomía, a saber: conocimiento, comprensión, aplicación, análisis, síntesis y evaluación (Bloom, Englehart, Furst, Hill y Krathwohl, 1956, citado en Halawi, Pires y McCarthy, 2009). En este sentido, el PMO entrega contenidos orientados secuencialmente para la adquisición progresiva de estos distintos horizontes de conocimiento, los cuales se articulan con actividades que permiten medir el logro del aprendizaje esperado. Tras la retroalimentación del tutor respecto al logro alcanzado, la respuesta se corrige o refuerza para así avanzar hacia un nivel más complejo de aprendizaje.

De esta forma, el PMO contempla un aprendizaje controlado y asincrónico, que integra la definición por objetivos que organizan la interacción entre alumno-material instruccional-tutor, a través de una estructurada programación de contenidos, para ser entregados de forma dosificada en el ciclo correspondiente. Asimismo, este aprendizaje por objetivos es también controlado, en cuanto se realiza un seguimiento constante de la efectiva adquisición de los conocimientos provistos, estandarizado para todas las asignaturas dictadas en el respectivo programa académico. Asincrónico, en cuanto los involucrados en el proceso formativo no concurren simultáneamente a la instancia educativa, sino que ésta es administrada en tiempos diferidos, pero coordinada sobre la base de determinadas tareas y plazos ${ }^{5}$.

El PMO se caracteriza por tener una estructura curricular dividida en ciclos de estudio, en los cuales se dictan dos asignaturas simultáneamente. Cada ciclo dura nueve semanas para los programas de pregrado y seis o siete para aquellos de posgrado, en tanto cada asignatura considera una carga horaria de noventa horas cronológicas, éstos son sucesivos, y dependerá del grado académico la cantidad de ciclos que componen la carrera en este modelo. Las asignaturas, por su parte, se estructuran según objetivos semanales, los cuales son específicos y secuenciados, y en la medida en que se avanza en el tiempo aumentan su dificultad. El cumplimiento de dichos objetivos es sistemáticamente evaluado mediante distintos mecanismos, tales

5 Director del Programa Modular Online UNIACC, entrevista personal, 17 de marzo de 2011. 
como: participación en foros de discusión semanal respecto de una pregunta de debate; resumen de los elementos centrales aprendidos por el alumno en la respectiva semana; controles de lectura aplicados semana por medio; trabajos individuales y grupales, y examen o trabajo final en la última semana. Estas distintas instancias donde se evalúa el desempeño académico tienen un puntaje asociado, el cual es integrado en un resultado final que se encuentra en una escala que va del uno al cien, la aprobación se consigue con sesenta o más puntos. Todo esto también soportado por la plataforma digital de Moodle.

Gráfico n. ${ }^{\circ}$ 2: Estructura curricular del modelo PMO

\begin{tabular}{|c|c|c|c|}
\hline $\begin{array}{l}\text { Ciclo } 1 \longmapsto \\
9 \text { semanas pregrado } \\
6 \text { semanas magíster }\end{array}$ & $\begin{array}{l}\text { Ciclo } 2 \longrightarrow \\
9 \text { semanas pregrado } \\
6 \text { semanas magíster }\end{array}$ & $\begin{array}{l}\text { Ciclo } 3 \longrightarrow \text { semanas pregrado } \\
6 \text { semanas magíster }\end{array}$ & Ciclos sucesivos \\
\hline $\begin{array}{c}\text { Asignatura } 1 \\
90 \mathrm{hrs} \text {. cronológicas }\end{array}$ & $\begin{array}{c}\text { Asignatura } 3 \\
90 \mathrm{hrs} \text {. cronológicas } \\
\end{array}$ & $\begin{array}{c}\text { Asignatura } 5 \\
90 \mathrm{hrs.} \text { cronológicas }\end{array}$ & $\begin{array}{l}\text { Hasta concluir la } \\
\text { totalidad de ciclos }\end{array}$ \\
\hline $\begin{array}{c}\text { Asignatura } 2 \\
90 \text { hrs. cronológicas }\end{array}$ & $\begin{array}{c}\text { Asignatura } 4 \\
90 \text { hrs. cronológicas }\end{array}$ & $\begin{array}{c}\text { Asignatura } 6 \\
90 \text { hrs. cronológicas }\end{array}$ & $\begin{array}{c}\text { cada carrera de pre y } \\
\text { posgrado }\end{array}$ \\
\hline
\end{tabular}

\section{Metodologías para evaluar programas e-learning}

La evaluación de un entorno educativo "intenta juntar y analizar o reflexionar sobre la experiencia o la evidencia disponible para decidir el valor de una intervención o actividad determinada" (Hodgson y Asensio, 2006: p. 53). Se orienta a desarrollar juicios de valor fundados sobre el proceso de enseñanza-aprendizaje, sobre la base de objetivos y metodologías acordes que se aplicarán en el estado inicial de dicho proceso (evaluación diagnóstica), durante este (evaluación formativa) o al final del mismo (evaluación sumativa) (Gutiérrez, 2003).

En cuanto a los objetivos de la evaluación, Hodgson y Watland (2004) mencionan específicamente, respecto al sistema e-learning, que gran parte de los estudios se ha focalizado en medir percepciones de los estudiantes para su comparación con el sistema presencial. Se considera la denominada evaluación actitudinal, que tal como evidencia Guitart (2002) en el concepto de actitud, remite a una tendencia psicológica que se expresa en la evaluación de un objeto específico, implicando reacciones de orden cognitivo, afectivo y 
conductual. De acuerdo a González Río (1997, citado en Rodríguez y Nieto, 2010, p. 177) una actitud se caracteriza por presentar los siguientes atributos:

- Dirección: que puede mostrar tendencia positiva o negativa hacia el objeto, en función si la valoración es favorable o desfavorable.

- Magnitud: que se manifiesta según el grado con que evalúa el objeto de actitud, bien sea a favor o en contra.

- Intensidad: asociada a la fuerza del sentimiento con la actitud.

- Centralidad: lo que supone interpretar la importancia de la actitud en la centralidad global del comportamiento del sujeto, implicando que a niveles más altos de centralidad en el sujeto, mayor resistencia al cambio.

De acuerdo a los autores antes mencionados, las evaluaciones actitudinales se orientan a estudiar las respuestas de los individuos ante determinados estímulos, a las cuales se les otorga una puntuación que permite diferenciar las disposiciones de éstos ante el objeto evaluado.

En una evaluación actitudinal de un programa e-learning, se deben considerar tanto los factores propios de cualquier actividad formativa como aquellos particulares de las posibilidades y requerimientos educativos del sistema, a saber: evaluación, institución, administración, diseño, contenidos y alumnos.

a) Institución: da cuenta de la confianza que le genera al alumno tanto la institución educacional, en términos de su expertise en materias de e-learning y de la propia disciplina de estudio, de sus respectivas autoridades y cuerpo docente, como los recursos e infraestructura que soportan el sistema a distancia (Van Slyke, Kittner y Belanger, 1998, citados en Rubio, 2003).

b) Administración: estima los elementos relativos al funcionamiento interno del programa, en cuanto a la relación estudiante-profesor y estudiante-recursos, la posibilidad y oportunidad de vinculación con ellos, asimismo el acceso al material requerido. Además, da cuenta sobre la facilidad de uso de la plataforma tecnológica y la utilización de todo su potencial en el contexto educativo (Berge, Collins y Dougherty, 2000, citado en Alvarado, 2003). 
c) Diseño: evalúa la planificación de la estructura curricular, es decir de cómo se organizan los contenidos que son provistos y cuál es su dosificación en el tiempo que dura el programa. En esta dimensión se considera también la claridad, lógica y pertinencia de dicha estructura, en relación a los contenidos y a la secuencia en que son provistos (Duart y Lupiáñez, 2004; McArdle, 2007).

d) Contenidos: considera específicamente en cada curso la perspectiva sobre los objetivos del proceso de enseñanza-aprendizaje, la necesidad formativa a satisfacer, la pertinencia de la orientación teórica de los cursos, la idoneidad del material producido y los propios logros del alumnado, es decir, la obtención de los contenidos esperados (Miller y Miller, 2000, citado en Alvarado, 2003; Duart y Lupiáñez, 2004). También se incluye el "traspaso de aprendizajes a contextos distintos al de adquisición" (Gutiérrez, 2003, p. 35), por tanto evidencia la aplicabilidad de los contenidos, es decir, de un aprendizaje significativo y útil para el quehacer de los alumnos.

e) Evaluación: vincula la necesidad de controlar sistemáticamente el progreso de los alumnos y el logro de los objetivos establecidos (Cánepa, 2005). Considera a su vez la idoneidad de las metodologías de evaluación de acuerdo a estos objetivos y a los recursos educativos entregados. Y por último, del conocimiento sobre qué, cómo y cuándo se evalúa, además de los criterios que se han utilizado para esto (Gutiérrez, 2003).

f) Alumnos: considera las características de los destinatarios de la formación, desde las variables sociodemográficas, conocimientos y habilidades individuales, hasta aspectos subjetivos de los alumnos como sus motivaciones y satisfacción (Van Slyke, Kittner y Belanger, 1998, citados en Rubio, 2003). En este estudio consideraremos los dos últimos.

\section{Objetivos}

\subsection{Objetivo general}

- Comparar las evaluaciones de los estudiantes respecto a diferentes factores de dos modelos de educación universitaria e-learning. 


\subsection{Objetivos específicos}

- Construir y validar una escala de evaluación de factores de educación e-learning.

- Determinar la significación estadística de las diferencias entre modelos e-learning respecto a seis diferentes factores levantados teóricamente.

\section{Marco metodológico}

\subsection{Hipótesis de trabajo}

Existirán diferencias significativas entre las evaluaciones de ambos grupos respecto al modelo pedagógico e-learning: los alumnos del modelo PMO evaluarán su modelo con valoraciones significativamente más altas.

\subsection{Tipo y diseño de investigación}

La presente investigación se enmarca dentro de los estudios no experimentales, comparativos inter grupos. Los grupos se definieron según modalidad de estudios, siendo los niveles de la variable "Programa Modular Online, PMO" y "Programa Especial de Titulación Online, PET Online".

\subsection{Muestra}

Las muestras de ambos grupos estuvieron constituidas de la siguiente manera:

Programa Modular Online, PMO:

Tamaño de la muestra: 57 personas entre 25 y 50 años.

Sexo: 30 hombres $(52,6 \%)$ y 27 mujeres $(47,4 \%)$.

Media de la edad: 34,77; desviación típica: 6,67.

Programa Especial de Titulación Online, PET Online:

Tamaño de la muestra: 104 personas entre 24 y 50 años.

Sexo: 56 hombres $(53,8 \%)$ y 48 mujeres $(46,2 \%)$.

Media de la edad: 36,79; desviación típica: 7,20. 
De manera tal que los cálculos que se hicieran fueran adecuados, vale decir, comparables en lo referente a "sexo" y "edad", se contrastaron ambos grupos respecto a dichas variables.

Con respecto a la variable "sexo", no se encontraron diferencias significativas entre ambos grupos, lo que habla de inexistencia de asociación entre "programa" y "sexo" (Chi cuadrado p $>0,05 ; \mathrm{V}$ de Cramer $\mathrm{p}>0,05)$.

En relación con la "edad", se examinaron los supuestos de normalidad, homocedasticidad e independencia. Luego de hacerlo, y por no cumplirse el supuesto de homocedasticidad, se optó por utilizar estadísticos de contraste no paramétricos. Al comparar, se observó que no habrían diferencias entre ambos grupos respecto a su "edad", con $U=2474,00 ; p>0,05$.

La distribución por programa de estudios, sexo y modalidad puede apreciarse en la tabla n. ${ }^{\circ} 1$.

Tabla n. ${ }^{\circ}$ 1: Muestra por programa académico, sexo y modalidad

\begin{tabular}{|l|c|c|c|c|}
\cline { 2 - 5 } \multicolumn{1}{c|}{} & \multicolumn{2}{c|}{ PMO } & \multicolumn{2}{c|}{ PET } \\
\hline Programa académico & Hombres & Mujeres & Hombres & Mujeres \\
\hline Psicología & 1 & 7 & 8 & 26 \\
\hline Ingeniería informática & 2 & 1 & 13 & 2 \\
\hline Ingeniería comercial & 8 & 0 & 20 & 13 \\
\hline Comunicación social & 2 & 5 & 6 & 0 \\
\hline Posgrado & 4 & 5 & 9 & 7 \\
\hline Bachillerato & 5 & 1 & 0 & 0 \\
\hline Licenciatura en administración pública & 8 & 8 & 0 & 0 \\
\hline Total & 30 & 27 & 56 & 48 \\
\hline
\end{tabular}

\subsection{Definición operacional de las variables}

a) Variable independiente: programa de estudios

Nivel de medición: nominal

Niveles de la variable:

- "PMO" (Programa Modular Online)

- "PET" (Programa Especial de Titulación Online) 
b) Variables dependientes: factores de educación online, definidos según revisión teórica.

Nivel de medición: intervalar (los ítems miden en formato Likert, siendo ordinales. Los factores son resultado del cálculo de la media de los diferentes ítems que pertenecen a dicho factor, siendo por ellos variables intervalares).

\subsection{Herramientas}

Escala ad hoc de medición de factores de educación e-learning: escala diseñada para evaluar seis diferentes factores relacionados con la apreciación de programas de estudios universitarios e-learning. Consta de treinta y tres ítems, en formato de afirmaciones positivas que el sujeto debe valorar en una escala Likert de 1 (totalmente en desacuerdo) a cinco (totalmente de acuerdo). Puede ser revisada en anexo.

Los seis factores son evaluados a través de los siguientes ítems:

Tabla n. ${ }^{\circ} 2$ : Ítems de evaluación por factor

\begin{tabular}{|l|c|}
\hline Factor según marco teórico & Preguntas \\
\hline Institución & $7,15,16,23,31$ \\
\hline Administración & $10,12,14,18,20,22,30$ \\
\hline Diseño & $1,4,5,13,17,26,29$ \\
\hline Contenido & $2,3,9,11,19,21,24,27$ \\
\hline Evaluación & $6,8,33$ \\
\hline Alumno & $25,28,32$ \\
\hline
\end{tabular}

\section{Resultados}

\subsection{Análisis de fiabilidad de las subescalas utilizadas para medir factores}

Los ítems que conformaron cada subescala para la medición de factores presentan coeficientes Alfa de Cronbach altos, tal como puede apreciarse en la tabla n. ${ }^{\circ} 3$. 
Tabla n. ${ }^{\circ}$ 3: Índice Alfa de Cronbach por factor

\begin{tabular}{|l|c|}
\hline Factor & Alfa de Cronbach \\
\hline Diseño & 0,885 \\
\hline Evaluación & 0,696 \\
\hline Administración & 0,866 \\
\hline Contenidos & 0,928 \\
\hline Alumnos & 0,733 \\
\hline Institución & 0,913 \\
\hline
\end{tabular}

\subsection{Comparación de factores}

Como se ha dicho, el estudio de los datos se basó en la comparación. Por no cumplirse el supuesto estadístico de normalidad en las distribuciones de las diferentes variables dependientes, se utilizaron pruebas de comparación no paramétricas.

Se compararon ambos grupos a partir de la variable independiente "programa de estudios", utilizando los factores como variables dependientes inter grupos.

En la mayoría de los casos pudo observarse medianas más altas en PMO que en PET. Sin embargo debieron realizarse pruebas estadísticas para verificar la significancia de los datos, de manera tal que se excluyeran resultados afectados por el azar.

Gráfico n. ${ }^{\circ}$ 3: Medianas comparadas PMO y PET por factor

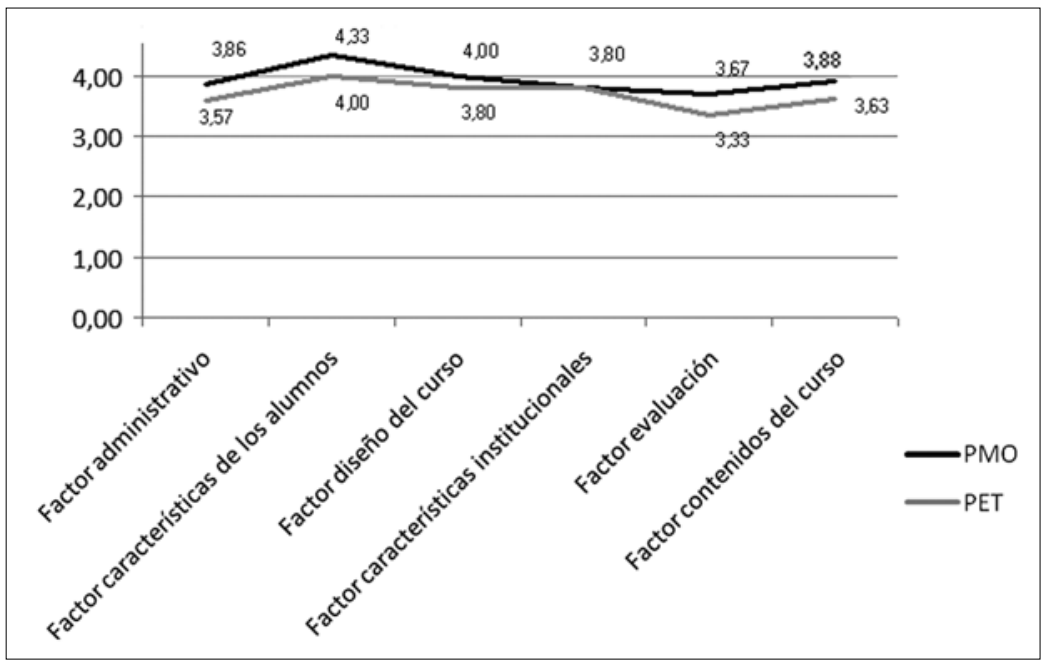


En el caso del factor "administración", la diferencia entre PMO y PET fue significativa $(U=2330,500 ; p<0,05)$, indicando que los puntajes del primero son efectivamente más altos. Lo mismo ocurre en el caso del factor "diseño" ( $U=2406,00 ; p<0,05)$, factor "evaluación" $(\mathrm{U}=2274,500 ; \mathrm{p}<0,05)$ y factor "contenidos" ( $U=2129,000 ; \mathrm{p}<0,01)$. En el caso de los factores características de los "alumnos" y de la "institución" no se encontraron diferencias significativas.

Se puede asentir respecto al factor "administración" que los alumnos evalúan mejor el PMO en términos del funcionamiento de la carrera, de la plataforma tecnológica y su idoneidad, como también la interacción con autoridades e instructores. Por su parte, sobre el factor "diseño", los alumnos del modelo PMO lo evalúan con valoraciones significativamente más altas sobre la conveniencia de la malla curricular, su claridad en cuanto a orden y lógica, y los respectivos objetivos establecidos. Dicha evaluación se condice con la del factor "contenidos", el que releva evaluaciones más favorables al PMO que al PET Online, sobre la pertinencia de los cursos, programas y objetivos expuestos, y el material de apoyo, para la consecuente transferencia de lo aprendido a su contexto de referencia. Respecto al factor "evaluación", los alumnos de programas en modalidad PMO, tienen una valoración más alta de aspectos como el monitoreo y seguimiento del desarrollo del proceso educativo, como específicamente la forma de evaluación y duración de la misma, que aquellos alumnos de programas en versión PET Online.

Respecto al factor "institución" y "características de los alumnos" no se encontraron diferencias significativas. Sobre la confianza en la institución y sus autoridades, como en los profesores y sus capacidades docentes, no hay distinción para los alumnos del PET Online y los del PMO. En tanto, la motivación y satisfacción global de los alumnos de dichas modalidades consideradas en el factor "características de los alumnos", tampoco se ven afectadas por la modalidad de los estudios.

Luego de realizar estos cálculos, observamos la distribución de las muestras según año de ingreso, tal como puede observarse en la tabla n. ${ }^{\circ} 4$. 
Tabla n. ${ }^{\circ}$ 4: Distribución de las muestras según año de ingreso

\begin{tabular}{|l|c|c|c|c|c|c|c|}
\hline & \multicolumn{7}{|c|}{ Año de ingreso } \\
\hline & 2005 & 2006 & 2007 & 2008 & 2009 & 2010 & Total \\
\hline PMO & 0 & 0 & 0 & 2 & 5 & 55 & 62 \\
\hline PET & 1 & 1 & 32 & 40 & 55 & 8 & 137 \\
\hline Total & 1 & 1 & 32 & 42 & 60 & 63 & 199 \\
\hline
\end{tabular}

Dado que podría pensarse que el programa de estudios (pregrado o posgrado) podría afectar los resultados, y si bien la muestra estuvo compuesta sólo por 25 alumnos de posgrado en total, comparamos ambos considerando sólo pregrado.

Al considerar sólo el pregrado para PET y PMO, se observaron similares diferencias en el factor "evaluación" ( $U=1619,500 ; p<0,05)$, "administración" ( $U=1682,500 ; \mathrm{p}<0,05)$ y "contenidos" ( $U=1523,000$; $\mathrm{p}<0,01)$. La significación de la diferencia en el factor "diseño" fue tendencial.

No se considera que la comparación entre alumnos de posgrado de PET (Muestra = 9) y PMO (muestra = 16) pudiese tener suficiente peso para hablar de real significación estadística en las conclusiones, dada la baja cantidad de muestra para cada una.

\section{Conclusión}

De los resultados antes detallados, se observa que tanto los alumnos del PET Online como los del PMO evalúan positivamente al concerniente sistema e-learning. No obstante, al comparar las evaluaciones de los diferentes factores de los dos modelos e-learning, se aprecia que los alumnos que siguieron estudios a través de la modalidad PMO, tienen una evaluación significativamente más alta del respectivo sistema que aquellos que estudiaron bajo la modalidad PET Online. Específicamente, la evaluación que se hace de los factores administración, diseño, contenido, y evaluación muestran diferencias significativamente mayores a favor de los programas PMO.

De acuerdo a estos resultados se puede sostener que la percepción favorable sobre el sistema educativo no se basa en el medio a través del cual se proveen los contenidos, que en este caso 
corresponde a la plataforma Moodle, tanto para el PET Online como para el PMO. Sino, es la estrategia pedagógica asociada a dicho medio la cual da cuenta de las diferencias a favor de un modelo con una estructurada programación de contenidos, dosificación en su entrega y actividades evaluativas que permiten orientar sistemáticamente todo el proceso de enseñanza-aprendizaje, potenciado con las posibilidades que entrega dicha plataforma digital.

El presente estudio contribuye en la aproximación empírica a las evaluaciones que usuarios del sistema e-learning pueden realizar, ratificando una percepción favorable de este medio. A su vez, la elaboración y aplicación de una escala ad hoc, constituye un referente como modelo evaluativo de los factores en éste considerados, sujeto a ser empleado en otros programas e-learning y a ser ampliado con nuevas variables. Consecuentemente, el estudio y divulgación de experiencias satisfactorias de e-learning aporta en la comprensión de este medio como facilitador de ambientes formativos, y entrega antecedentes que pueden ser utilizados para sustentar innovaciones en estas materias, y avanzar sistemáticamente hacia sistemas cada vez más efectivos y pertinentes a los fines de la educación.

Dado que la muestra estuvo compuesta exclusivamente por estudiantes de estos dos modelos, sería valioso considerar extender la investigación hacia otros actores relevantes implicados en el sistema de educación, y a quienes se ven impactados por el mismo. Asimismo, incorporar otras instituciones y modelos pedagógicos que podría aportar hacia una comprensión más extensiva del fenómeno del e-learning en Chile. En consecuencia, continuar hacia una comprensión de las tecnologías de la información y comunicación, en función de su carácter instrumental en el contexto didáctico, posibilitando mejores mecanismos para el desarrollo de instancias de enseñanza-aprendizaje, las cuales son el eje de la educación.

\section{Referencias bibliográficas}

Allen, I. E. y Seaman, J. (2010) Class Differences. Online Education in the United States, 2010. Babson Survey Research Group \& Sloan Consortium. Disponible en http://sloanconsortium.org/publications/survey/pdf/ class_differences.pdf 
Alvarado, A. (2003) Diseño instruccional para la producción de cursos en línea y e-learning. Revista Docencia Universitaria, 4 (1), pp. 9-24.

Cabrera, L. (2009) Universidad a distancia de UNIACC. Ponencia dictada en la IV Jornada Tecnológica de la Universidad Austral de Chile, Valdivia.

Cánepa, C. (2005) Marco conceptual para la construcción de un modelo de e-learning. Revista de Investigación en Sistemas e Informática, 2 (2), pp. 69-77.

Duart, J. y Lupiáñez, F. (2004) Procesos institucionales de gestión de la calidad del e-learning en instituciones educativas universitarias. Boletín Electrónico del Viceministerio de Educación Superior. Bogotá. Disponible en www.mineducacion.gov.co/1621/articles-85679_Archivo_pdf.pdf

Guitart, R. (2002) Las actitudes en el centro escolar. Reflexiones y propuestas. Barcelona: Graó.

Gutiérrez, O. A. (2003) Alternativas en la evaluación de los aprendizajes. La evaluación en los enfoques centrados en el aprendizaje. Disponible en http:// www.lie.upn.mx/docs/docinteres/EnfoquesyModelosEducativos4.pdf

Halawi, L. A., Pires, S. y McCarthy, R. V. (2009) An evaluation of e-learning on the basis of Bloom's Taxonomy: An exploratory study. Journal of Education for Business, 84 (6), pp. 374-380. Disponible en ProQuest Education Journals.

Hodgson, V. y Ascencio, M. (2006) La evaluación en la educación virtual. Memorias de la $1^{a}$ Conferencia Internacional ELAC (tecnologías de comunicación e información para la educación superior). México. Disponible en http://www.elacvirtual.net/documents/conferencias_ elac/I_conferencia/6_Vivienmireia.pdf

Hodgson, V. y Watland, P. (2004) Researching networked management learning. Management Learning, 35, pp. 99-116. doi: 10.1177/1350507604043027.

McArdle, G. E. (2007) Training design and delivery ( ${ }^{\text {nd }}$ Ed.). Alexandria, VA: American Society for Training and Development Press.

Riquelme, P. y Martínez, C. (2008) Estado de la educación superior online. Disponible en http://productosonline.files.wordpress. com/2008/08/200802-estado-eol-chile.pdf

Rodríguez, M. J. y Nieto, S. (Coords.) (2010) Investigación y evaluación educativa en la sociedad del conocimiento. Salamanca: Ediciones Universidad de Salamanca.

Rubio, M. J. (2003) Enfoques y modelos de evaluación del e-learning. Revista Electrónica de Investigación y Evaluación Educativa, RELIEVE, 9 (2), pp. 101-120. Disponible en http://www.uv.es/RELIEVE/v9n2/ RELIEVEv9n2_1.htm 
120 INVESTIGACIÓN COMPARATIVA SOBRE LAS EVALUACIONES DE ESTUDIANTES UNIVERSITARIOS FRENTE A DOS MODELOS E-LEARNING - A. Mendiburo-Seguel, M. Reininger

Simonson, M., Smaldino, S., Albright, M. y Zvacek, S. (2006) Teaching and learning at a distance: Foundations of distance education ( $3^{\mathrm{a}} \mathrm{Ed}$.). Upper Saddle River, NJ: Merrill Prentice Hall.

Universidad Virtual-REUNA (2003). La educación virtual en Chile: Historia, estado del arte y proyecciones. Hacia el conocimiento de la realidad nacional en materia de uso de tecnología aplicada a la educación superior. Disponible en http://unesdoc.unesco.org/images/0014/001403/140393s.pdf

Recibido: 05/04/2011

Aceptado 23/05/2011 


\section{Anexo: Escala de medición de factores de la educación on-line}

A continuación encontrará una serie de afirmaciones respecto a la carrera que estudia. Cada una de ellas es evaluable respecto a su grado de acuerdo (desde "totalmente en desacuerdo" hasta "totalmente de acuerdo". Por favor, marque cada una de ellas dependiendo de su opinión.

Garantizamos su total anonimato, dado que lo que nos interesa es conocer la opinión del total de encuestados y no la información individual.

SEXO: $\quad$ M $F$

EDAD:

CARRERA:

MODALIDAD:

AÑO:

\begin{tabular}{|c|c|c|c|c|c|c|}
\hline & La duración de los cursos es adecuada. & $\begin{array}{c}\text { Totalmente en } \\
\text { desacuerdo }\end{array}$ & $\begin{array}{c}\text { En } \\
\text { desacuerdo } \\
\end{array}$ & $\begin{array}{l}\text { Ni de acuerdo ni } \\
\text { en desacuerdo }\end{array}$ & $\begin{array}{c}\text { De } \\
\text { acuerdo }\end{array}$ & $\begin{array}{l}\text { Totalmente } \\
\text { de acuerdo }\end{array}$ \\
\hline & $\begin{array}{l}\text { El diseño de la malla curricular me ha } \\
\text { permitido aplicar en mi vida cotidiana lo } \\
\text { que he aprendido en los cursos. }\end{array}$ & $\begin{array}{c}\text { Totalmente en } \\
\text { desacuerdo }\end{array}$ & $\begin{array}{c}\text { En } \\
\text { desacuerdo }\end{array}$ & $\begin{array}{l}\text { Ni de acuerdo ni } \\
\text { en desacuerdo }\end{array}$ & $\begin{array}{l}\text { De } \\
\text { acuerdo }\end{array}$ & $\begin{array}{l}\text { Totalmente } \\
\text { de acuerdo }\end{array}$ \\
\hline 3 & Los contenidos de los cursos son claros. & $\begin{array}{c}\text { Totalmente en } \\
\text { desacuerdo }\end{array}$ & \begin{tabular}{|c|} 
En \\
desacuerdo
\end{tabular} & $\begin{array}{l}\text { Ni de acuerdo ni } \\
\text { en desacuerdo }\end{array}$ & $\begin{array}{c}\text { De } \\
\text { acuerdo }\end{array}$ & $\begin{array}{l}\text { Totalmente } \\
\text { de acuerdo }\end{array}$ \\
\hline 4 & $\begin{array}{l}\text { Considero que he aprendido mucho } \\
\text { gracias a la malla curricular. }\end{array}$ & $\begin{array}{c}\text { Totalmente en } \\
\text { desacuerdo }\end{array}$ & $\begin{array}{c}\text { En } \\
\text { desacuerdo }\end{array}$ & $\begin{array}{l}\text { Ni de acuerdo ni } \\
\text { en desacuerdo }\end{array}$ & $\begin{array}{c}\text { De } \\
\text { acuerdo }\end{array}$ & $\begin{array}{l}\text { Totalmente } \\
\text { de acuerdo }\end{array}$ \\
\hline 5 & $\begin{array}{l}\text { La malla curricular cumple con sus } \\
\text { objetivos. }\end{array}$ & $\begin{array}{c}\text { Totalmente en } \\
\text { desacuerdo }\end{array}$ & $\begin{array}{c}\text { En } \\
\text { desacuerdo }\end{array}$ & $\begin{array}{l}\text { Ni de ac } \\
\text { en des: }\end{array}$ & $\begin{array}{c}\text { De } \\
\text { acuerdo }\end{array}$ & $\begin{array}{l}\text { nente } \\
\text { uerdo }\end{array}$ \\
\hline 6 & $\begin{array}{l}\text { La forma de evaluación de las cátedras } \\
\text { es adecuada. }\end{array}$ & $\begin{array}{c}\text { Totalmente en } \\
\text { desacuerdo }\end{array}$ & \begin{tabular}{|c|} 
En \\
desacuerdo
\end{tabular} & $\begin{array}{l}\text { Ni de ac } \\
\text { en des }\end{array}$ & $\begin{array}{c}\text { De } \\
\text { acuerdo }\end{array}$ & $\begin{array}{l}\text { nente } \\
\text { lerdo }\end{array}$ \\
\hline 7 & $\begin{array}{l}\text { Por lo que he vivido en la carrera, } \\
\text { UNIACC me parece confiable. }\end{array}$ & $\begin{array}{c}\text { Totalmente en } \\
\text { desacuerdo }\end{array}$ & \begin{tabular}{|c|} 
En \\
desacuerdo
\end{tabular} & $\begin{array}{l}\text { Ni de a } \\
\text { en de }\end{array}$ & $\begin{array}{c}\text { De } \\
\text { acuerdo }\end{array}$ & \\
\hline 8 & $\begin{array}{l}\text { La duración de las evaluaciones es } \\
\text { adecuada. }\end{array}$ & $\begin{array}{c}\text { Totalmente en } \\
\text { desacuerdo }\end{array}$ & \begin{tabular}{|c|} 
En \\
desacuerdo
\end{tabular} & $\begin{array}{l}\text { Ni de a } \\
\text { en de }\end{array}$ & \begin{tabular}{|c} 
De \\
acuerdo
\end{tabular} & \\
\hline 9 & Los cursos cumplen con sus objetivos. & $\begin{array}{r}\text { Totalme } \\
\text { desact }\end{array}$ & \begin{tabular}{|c|} 
En \\
desacuerdo
\end{tabular} & $\begin{array}{l}\text { Ni de a } \\
\text { en de }\end{array}$ & $\begin{array}{c}\text { De } \\
\text { acuerdo }\end{array}$ & \\
\hline & $\begin{array}{l}\text { La carre } \\
\text { fechas C }\end{array}$ & $\begin{array}{r}\text { Totalm } \\
\text { desac }\end{array}$ & $\begin{array}{c}\text { En } \\
\text { desacuerdo }\end{array}$ & $\begin{array}{l}\text { Ni de } \\
\text { en de }\end{array}$ & \begin{tabular}{|c} 
De \\
acuerdo
\end{tabular} & \\
\hline 11 & Los programas de los cursos son claros. & $\begin{array}{r}\text { Totalm } \\
\text { desac }\end{array}$ & \begin{tabular}{|c|} 
En \\
desacuerdo
\end{tabular} & $\begin{array}{l}\text { Ni de a } \\
\text { en de }\end{array}$ & $\begin{array}{c}\text { De } \\
\text { acuerdo }\end{array}$ & \\
\hline 12 & actarse con las autoridades & $\begin{array}{r}\text { Totalme } \\
\text { desacu }\end{array}$ & $\begin{array}{c}\text { En } \\
\text { desacuerdo }\end{array}$ & $\begin{array}{l}\text { Ni de } \\
\text { en de }\end{array}$ & $\begin{array}{c}\text { De } \\
\text { acuerdo }\end{array}$ & \\
\hline 13 & $\begin{array}{l}\text { Al entrar a la carrera se tiene total } \\
\text { claridad sobre la malla curricular. }\end{array}$ & $\begin{array}{c}\text { Totalmente en } \\
\text { desacuerdo }\end{array}$ & $\begin{array}{c}\text { En } \\
\text { desacuerdo }\end{array}$ & $\begin{array}{l}\text { cerdo ni } \\
\text { cuerdo }\end{array}$ & $\begin{array}{c}\text { De } \\
\text { acuerdo }\end{array}$ & \\
\hline 14 & $\begin{array}{l}\text { El funcionamiento de la carrera es } \\
\text { bueno. }\end{array}$ & $\begin{array}{c}\text { Totalmente en } \\
\text { desacuerdo }\end{array}$ & \begin{tabular}{|c|} 
En \\
desacuerdo
\end{tabular} & $\begin{array}{l}\text { Ni de al } \\
\text { en des }\end{array}$ & \begin{tabular}{|c} 
De \\
acuerdo
\end{tabular} & $\begin{array}{l}\text { Totalmente } \\
\text { de acuerdo }\end{array}$ \\
\hline 15 & $\begin{array}{l}\text { Por lo que he vivido en la carrera, las } \\
\text { autoridades de UNIACC me parecen } \\
\text { confiables. }\end{array}$ & $\begin{array}{c}\text { Totalmente en } \\
\text { desacuerdo }\end{array}$ & $\begin{array}{c}\text { En } \\
\text { desacuerdo }\end{array}$ & $\begin{array}{l}\text { Ni de acuerdo ni } \\
\text { en desacuerdo }\end{array}$ & $\begin{array}{c}\text { De } \\
\text { acuerdo }\end{array}$ & $\begin{array}{l}\text { Totalmente } \\
\text { de acuerdo }\end{array}$ \\
\hline & $\begin{array}{l}\text { Los docentes están capacitados para } \\
\text { enseñar. }\end{array}$ & $\begin{array}{c}\text { Totalmente en } \\
\text { desacuerdo }\end{array}$ & \begin{tabular}{|c|} 
En \\
desacuerdo
\end{tabular} & $\begin{array}{l}\text { Ni de acuerdo ni } \\
\text { en desacuerdo }\end{array}$ & \begin{tabular}{|c} 
De \\
acuerdo
\end{tabular} & $\begin{array}{l}\text { Totalmente } \\
\text { de acuerdo }\end{array}$ \\
\hline
\end{tabular}


UNIVERSITARIOS FRENTE A DOS MODELOS E-LEARNING - A. Mendiburo-Seguel, M. Reininger

\begin{tabular}{|c|c|c|c|c|c|c|}
\hline & $\begin{array}{l}\text { El orden de las cátedras en la carrera } \\
\text { sigue una lógica clara. }\end{array}$ & \begin{tabular}{|c|}
$\begin{array}{c}\text { Totalmente en } \\
\text { desacuerdo }\end{array}$ \\
\end{tabular} & \begin{tabular}{|c|} 
En \\
desacuerdo
\end{tabular} & \begin{tabular}{|c|} 
Ni de acuerdo ni \\
en desacuerdo
\end{tabular} & \begin{tabular}{|c|} 
De \\
acuerdo
\end{tabular} & $\begin{array}{l}\text { Totalmente } \\
\text { de acuerdo }\end{array}$ \\
\hline & $\begin{array}{l}\text { Es fácil interactuar con los instructores } \\
\text { de los cursos. }\end{array}$ & $\begin{array}{c}\text { Totalmente en } \\
\text { desacuerdo }\end{array}$ & $\begin{array}{c}\text { En } \\
\text { desacuerdo }\end{array}$ & $\begin{array}{c}\text { Ni de acuerdo ni } \\
\text { en desacuerdo }\end{array}$ & $\begin{array}{c}\text { De } \\
\text { acuerdo }\end{array}$ & $\begin{array}{l}\text { Totalmente } \\
\text { de acuerdo }\end{array}$ \\
\hline & $\begin{array}{l}\text { Los contenidos de los cursos son } \\
\text { pertinentes. }\end{array}$ & $\begin{array}{c}\text { Totalmente en } \\
\text { desacuerdo }\end{array}$ & \begin{tabular}{|c|} 
En \\
desacuerdo
\end{tabular} & $\begin{array}{c}\text { Ni de acuerdo ni } \\
\text { en desacuerdo }\end{array}$ & $\begin{array}{c}\text { De } \\
\text { acuerdo }\end{array}$ & $\begin{array}{l}\text { Totalmente } \\
\text { de acuerdo }\end{array}$ \\
\hline 20 & $\begin{array}{l}\text { La plataforma tecnológica que soporta el } \\
\text { curso funciona bien. }\end{array}$ & $\begin{array}{c}\text { Totalmente en } \\
\text { desacuerdo }\end{array}$ & \begin{tabular}{|c|} 
En \\
desacuerdo
\end{tabular} & \begin{tabular}{|c|}
$\begin{array}{c}\text { Ni de acuerdo ni } \\
\text { en desacuerdo }\end{array}$ \\
\end{tabular} & $\begin{array}{c}\text { De } \\
\text { acuerdo }\end{array}$ & $\begin{array}{l}\text { Totalmente } \\
\text { de acuerdo }\end{array}$ \\
\hline 21 & $\begin{array}{l}\text { Las lecturas y textos del curso son } \\
\text { adecuados. }\end{array}$ & $\begin{array}{c}\text { Totalmente en } \\
\text { desacuerdo }\end{array}$ & $\begin{array}{c}\text { En } \\
\text { desacuerdo }\end{array}$ & $\begin{array}{c}\text { Ni de acuerdo ni } \\
\text { en desacuerdo }\end{array}$ & $\begin{array}{c}\text { De } \\
\text { acuerdo }\end{array}$ & $\begin{array}{l}\text { Totalmente } \\
\text { de acuerdo }\end{array}$ \\
\hline 22 & $\begin{array}{l}\text { La plataforma tecnológica que soporta el } \\
\text { curso permite que aprender ser más fácil. }\end{array}$ & $\begin{array}{c}\text { Totalmente en } \\
\text { desacuerdo }\end{array}$ & $\begin{array}{c}\text { En } \\
\text { desacuerdo }\end{array}$ & \begin{tabular}{|c|} 
Ni de acuerdo ni \\
en desacuerdo
\end{tabular} & $\begin{array}{c}\text { De } \\
\text { acuerdo }\end{array}$ & $\begin{array}{l}\text { Totalmente } \\
\text { de acuerdo }\end{array}$ \\
\hline 23 & $\begin{array}{l}\text { Considero que he aprendido mucho } \\
\text { gracias al manejo de los docentes. }\end{array}$ & $\begin{array}{c}\begin{array}{c}\text { Totalmente en } \\
\text { desacuerdo }\end{array} \\
\end{array}$ & $\begin{array}{c}\text { En } \\
\text { desacuerdo }\end{array}$ & $\begin{array}{c}\text { Ni de acuerdo ni } \\
\text { en desacuerdo }\end{array}$ & $\begin{array}{c}\text { De } \\
\text { acuerdo }\end{array}$ & $\begin{array}{l}\text { Totalmente } \\
\text { de acuerdo }\end{array}$ \\
\hline 24 & $\begin{array}{l}\text { La orientación teórica de los cursos que } \\
\text { forman la malla curricular es adecuada. }\end{array}$ & $\begin{array}{c}\text { Totalmente en } \\
\text { desacuerdo }\end{array}$ & $\begin{array}{c}\text { En } \\
\text { desacuerdo }\end{array}$ & \begin{tabular}{|c|} 
Ni de acuerdo ni \\
en desacuerdo
\end{tabular} & $\begin{array}{c}\text { De } \\
\text { acuerdo }\end{array}$ & $\begin{array}{l}\text { Totalmente } \\
\text { de acuerdo }\end{array}$ \\
\hline & $\begin{array}{l}\text { Al comenzar la carrera me sentía } \\
\text { motivado por estudiarla. }\end{array}$ & $\begin{array}{c}\text { Totalmente en } \\
\text { desacuerdo }\end{array}$ & \begin{tabular}{|c|} 
En \\
desacuerdo
\end{tabular} & $\begin{array}{c}\text { Ni de acuerdo ni } \\
\text { en desacuerdo }\end{array}$ & $\begin{array}{c}\text { De } \\
\text { acuerdo }\end{array}$ & $\begin{array}{l}\text { Totalmente } \\
\text { de acuerdo }\end{array}$ \\
\hline & $\begin{array}{l}\text { La estructura de la malla curricular es } \\
\text { adecuada. }\end{array}$ & $\begin{array}{c}\text { Totalmente en } \\
\text { desacuerdo }\end{array}$ & $\begin{array}{c}\text { En } \\
\text { desacuerdo }\end{array}$ & $\begin{array}{c}\text { Ni de acuerdo ni } \\
\text { en desacuerdo }\end{array}$ & $\begin{array}{c}\text { De } \\
\text { acuerdo }\end{array}$ & $\begin{array}{l}\text { Totalmente } \\
\text { de acuerdo }\end{array}$ \\
\hline 27 & $\begin{array}{l}\text { Se utiliza material audiovisual de buena } \\
\text { manera. }\end{array}$ & $\begin{array}{c}\text { Totalmente en } \\
\text { desacuerdo }\end{array}$ & $\begin{array}{c}\text { En } \\
\text { desacuerdo }\end{array}$ & $\begin{array}{c}\text { Ni de acuerdo ni } \\
\text { en desacuerdo }\end{array}$ & $\begin{array}{c}\text { De } \\
\text { acuerdo }\end{array}$ & $\begin{array}{l}\text { Totalmente } \\
\text { de acuerdo }\end{array}$ \\
\hline & $\begin{array}{l}\text { En lo global, estoy satisfecho con la } \\
\text { carrera. }\end{array}$ & $\begin{array}{c}\text { Totalmente en } \\
\text { desacuerdo }\end{array}$ & $\begin{array}{c}\text { En } \\
\text { desacuerdo }\end{array}$ & $\begin{array}{c}\text { Ni de acuerdo ni } \\
\text { en desacuerdo }\end{array}$ & $\begin{array}{c}\text { De } \\
\text { acuerdo }\end{array}$ & $\begin{array}{l}\text { Totalmente } \\
\text { de acuerdo }\end{array}$ \\
\hline & $\begin{array}{l}\text { Los cursos de la malla curricular se } \\
\text { suceden de manera adecuada. }\end{array}$ & $\begin{array}{c}\text { Totalmente en } \\
\text { desacuerdo }\end{array}$ & $\begin{array}{c}\text { En } \\
\text { desacuerdo }\end{array}$ & $\begin{array}{c}\text { Ni de acuerdo ni } \\
\text { en desacuerdo }\end{array}$ & $\begin{array}{c}\text { De } \\
\text { acuerdo }\end{array}$ & $\begin{array}{l}\text { Totalmente } \\
\text { de acuerdo }\end{array}$ \\
\hline 30 & $\begin{array}{l}\text { Existe sinergia en el proceso de } \\
\text { enseñanza-aprendizaje entre los } \\
\text { estudiantes y los docentes. }\end{array}$ & $\begin{array}{c}\text { Totalmente en } \\
\text { desacuerdo }\end{array}$ & \begin{tabular}{|c|} 
En \\
desacuerdo
\end{tabular} & $\begin{array}{c}\text { Ni de acuerdo ni } \\
\text { en desacuerdo }\end{array}$ & $\begin{array}{c}\text { De } \\
\text { acuerdo }\end{array}$ & $\begin{array}{l}\text { Totalmente } \\
\text { de acuerdo }\end{array}$ \\
\hline 31 & $\begin{array}{l}\text { Por lo que he vivido en la carrera, } \\
\text { los docentes de UNIACC me parecen } \\
\text { confiables. }\end{array}$ & \begin{tabular}{|c|}
$\begin{array}{c}\text { Totalmente en } \\
\text { desacuerdo }\end{array}$ \\
\end{tabular} & \begin{tabular}{|c|} 
En \\
desacuerdo
\end{tabular} & $\begin{array}{c}\text { Ni de acuerdo ni } \\
\text { en desacuerdo }\end{array}$ & \begin{tabular}{|c|} 
De \\
acuerdo
\end{tabular} & $\begin{array}{l}\text { Totalmente } \\
\text { de acuerdo }\end{array}$ \\
\hline 32 & $\begin{array}{l}\text { Luego del tiempo que llevo de estudio, } \\
\text { me siento motivado por la carrera. }\end{array}$ & \begin{tabular}{|c|}
$\begin{array}{c}\text { Totalmente en } \\
\text { desacuerdo }\end{array}$ \\
\end{tabular} & \begin{tabular}{|c|} 
En \\
desacuerdo
\end{tabular} & \begin{tabular}{|c|} 
Ni de acuerdo ni \\
en desacuerdo
\end{tabular} & $\begin{array}{c}\text { De } \\
\text { acuerdo }\end{array}$ & $\begin{array}{l}\text { Totalmente } \\
\text { de acuerdo }\end{array}$ \\
\hline & $\begin{array}{l}\text { Se hace un monitoreo y seguimiento } \\
\text { de los estudiantes por parte de las } \\
\text { autoridades de la carrera. }\end{array}$ & $\begin{array}{c}\text { Totalmente en } \\
\text { desacuerdo }\end{array}$ & $\begin{array}{c}\text { En } \\
\text { desacuerdo }\end{array}$ & $\begin{array}{c}\text { Ni de acuerdo ni } \\
\text { en desacuerdo }\end{array}$ & $\begin{array}{c}\text { De } \\
\text { acuerdo }\end{array}$ & $\begin{array}{l}\text { Totalmente } \\
\text { de acuerdo }\end{array}$ \\
\hline
\end{tabular}

